\title{
DIFFUSE C IV EMISSION AROUND THE WO STAR ST3
}

V.F. Polcaro(1,2), F.Giovannelli (1), R.K. Manchanda(3),

A. Pollock (4), L. Norci(5,6), C. Rossi(6)

1) Istituto di Astrofisica Spaziale, CNR, Frascati, Italy

2) Dip. Aerospaziale, Univ. "La Sapienza", Rome, Italy

3) Tata Institute of Fundamental Research, Bombay, India

4) Space Science Departement of ESA, Noordwijck, The Netherlands

5) Max-Plank-Institut fur Extraterrestrische Physik, Garching, FRG

6) Istituto di Astronomia, Univ. "La Sapienza", Rome, Italy

A strong diffuse C IV (5801-12 A) emission has been discovered in Berkeley 87 cluster near to the WR (WO) star ST3 (Barlow and Hummer, 1982) using the Loiano $152 \mathrm{~cm}$ telescope equipped with a Boller and Chivens 26767 spectrograph and a CCD RCA camera. The diffuse emission seems to interest all the core of the cluster Berkeley 87. In fact it is present around many cluster members sited near to the center.

This young open cluster is embedded in the ON2 molecular clouds complex on the boundary of the Cygnus X region (Turner and Forbes, 1982). A weak hard $\mathrm{X}$-ray source, positionally coincident with the cluster, was identified during a slew by EXOSAT satellite (Warwick et al., 1988). We can thus suppose that the envelope of ST3 is strongly interacting with the cluster interstellar medium.

The C IV diffuse line should originate from the shock- ionized material at the interacting boundary between the wind (our measurements give a $5200 \mathrm{Km} / \mathrm{s}$ velocity from the Doppler broadening of the He II 6561 line) and the molecular clouds present in the cluster. The flux in the diffuse line can be evaluated to be at least the 45star at the same wavelength. Given that the C IV is the only carbon ion present in the star spectrum, the classification of ST 3 as WC star can be uncertain.

\section{References}

Barlow M.J. and Hummer, D.C., 1982: Proc of the IAU Symposium on "Wolf-Rayet Stars: Observation, Physics, Evolution", C. de Loore and A. Willis eds, p.387.

Turner, D.G. AND Forbes, D., 1982: Publ. Astr. Soc. Pac., 94, 789.

Warwick, R. S., Norton, A. J., Turner, M. J. L., Watson, M. G., Willingale, R., 1988: Mon. Not. R. astr. Soc. 232, 551. 
104

Fig. 1: a) Low dispersion spectrum of ST 3 between 4000-7100 A, taken at the Loiano Telescope on August 31, 1989 (detector CCD RCA coated, exposure time $1000 \mathrm{~s})$.

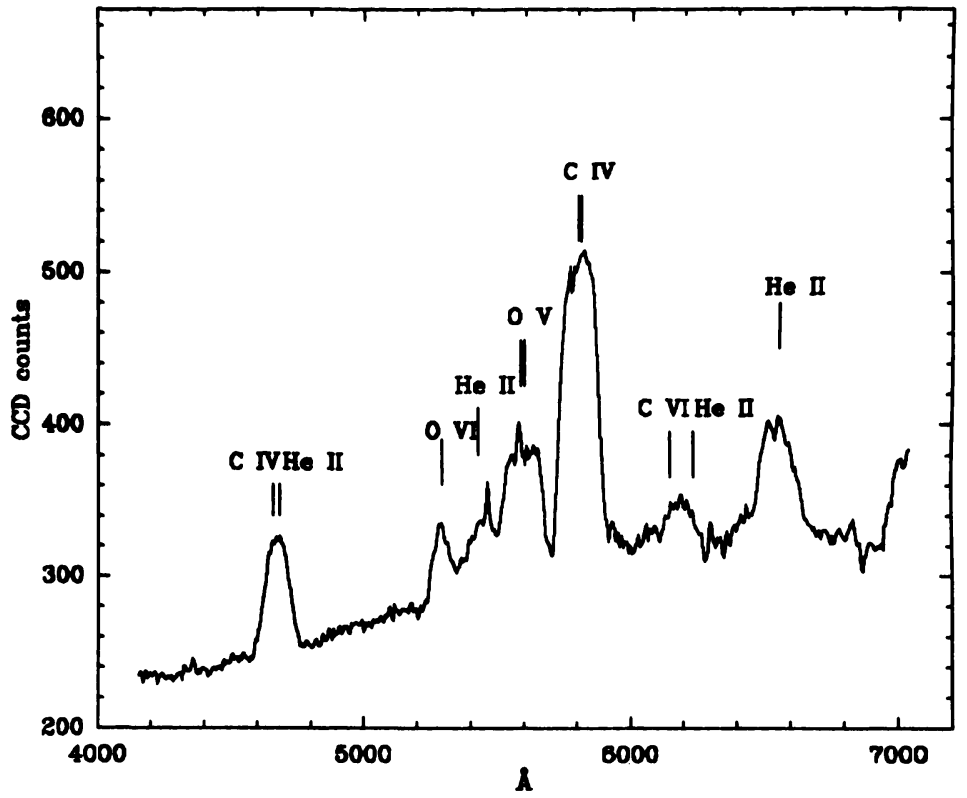

Fig. 2: The sky spectrum around (a) ST3 and (b) the standard star BD+28 4211. Local sky features and the diffuse emission are marked.

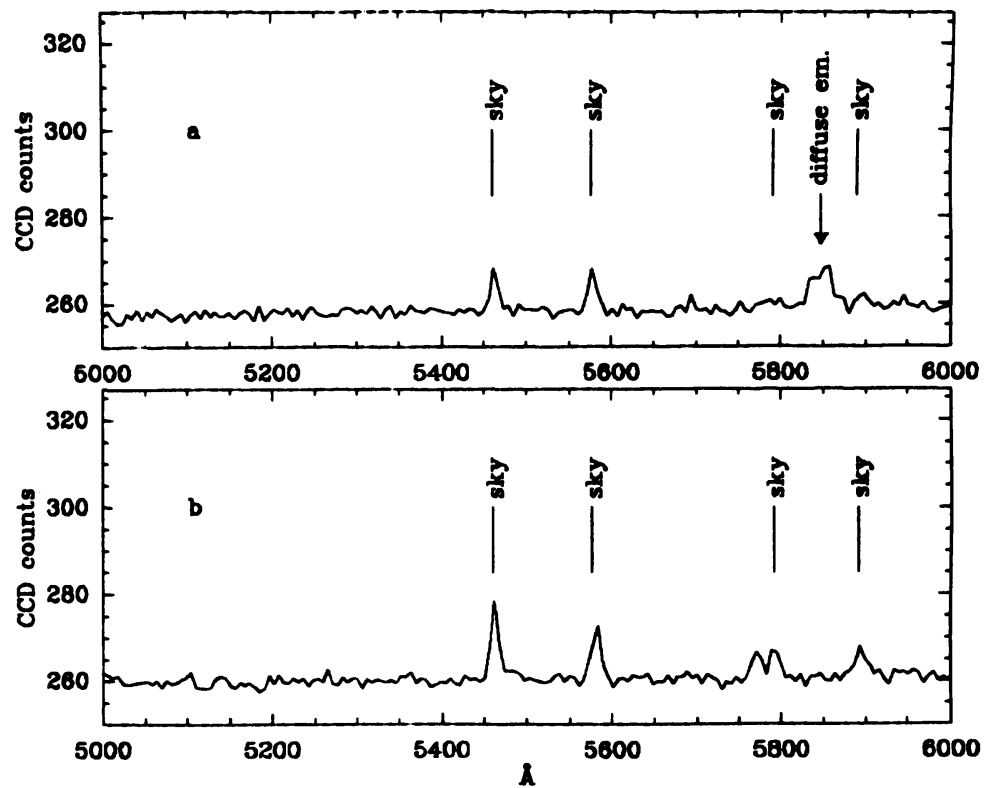

